Pacific

Journal of

Mathematics

\title{
A TOPOLOGICAL SPHERE THEOREM FOR
} ARBITRARY-DIMENSIONAL MANIFOLDS

YULIANG Wen 


\title{
A TOPOLOGICAL SPHERE THEOREM FOR ARBITRARY-DIMENSIONAL MANIFOLDS
}

\author{
YULIANG WEN
}

\begin{abstract}
We study manifolds with bounded volume, sectional curvature, and injectivity radius. We obtain a topological sphere theorem.
\end{abstract}

Sphere theorems are common in differential geometry; one often asks whether a manifold is homeomorphic to a sphere under certain topological or geometric restrictions; see for instance [Grove and Shiohama 1977; Perelman 1995; Shen 1989; Shiohama 1983; Suyama 1991; Wu 1989]. Coghlan and Itokawa [1991] proved a sphere theorem that says that if an even-dimensional, simply connected Riemannian manifold $\mathcal{M}$ has sectional curvature $K_{\mathcal{M}} \in(0,1]$, volume $V_{\mathcal{M}} \leq \frac{3}{2} V_{S^{n}}$ with $V_{S^{n}}$ the volume of the standard $n$-dimensional unit sphere $S^{n}$ in $\mathbb{R}^{n+1}$, then $M$ must be homeomorphic to $S^{n}$. In [Wen 2004], we improved this result by relaxing the upper bound on $V_{\mathcal{M}}$ to a bound larger than $\frac{3}{2} V_{S^{n}}$. In both of these papers, the hypotheses of simple connectivity and even dimension were merely used to deduce that the injectivity radius $i_{\mathcal{M}}$ is no less than $\pi$. Here we find that we can weaken the assumptions on $K_{\mathcal{M}}$ and $i_{\mathcal{M}}$. If the simple connectivity condition is removed, the conclusion holds in any dimension.

Before stating our result, we introduce some notation. Let $(\mathcal{M}, g)$ be a compact, connected $n$-dimensional Riemannian manifold with metric $g$. We denote by $K_{\mathcal{M}}$ the sectional curvature of $\mathcal{M}$, by $i_{\mathcal{M}}$ its injectivity radius, and by $V_{\mathcal{M}}$ its volume. For any points $P, Q \in M$, we denote by $\gamma_{P, Q}$ the shortest geodesic on $M$ from $P$ to $Q$. Theorem 1. Given $k>0$, there exists an $\varepsilon_{0}>0$ such that if a compact connected $n$-dimensional Riemannian manifold $(\mathcal{M}, g)$ satisfies

$$
-k^{2} \leq K_{\mathcal{M}} \leq 1, \quad i_{M} \geq \pi-\varepsilon_{0}, \quad V_{\mu} \leq \frac{3}{2} V_{s^{n}}+\varepsilon_{0},
$$

then $\mathcal{M}$ is homeomorphic to $S^{n}$.

The examples of real projective spaces $\mathbb{R} P^{n}$ for $n \geq 2$ and product manifolds $S^{n} \times S^{m}$ for $m, n \geq 1$ show that the hypotheses on the lower bound on $i_{\mathcal{M}}$ or the upper bound on $V_{\mathcal{M}}$ cannot be removed.

MSC2000: 53C20.

Keywords: sphere theorem.

Supported in part by National Science Foundation of China (number 10871069) and Shanghai Leading Academic Discipline Project (project number B407). 
In what follows, we denote by $\mathbb{B}(P, r)$ the open geodesic ball in $M$ with center $P$ and radius $r$, and by $\overline{\mathbb{B}}(P, r)$ its closure. Also, we denote by $\mathscr{P}_{r}$ the open geodesic ball in $S^{n}$ with radius $r$. Instead of proving Theorem 1 directly, we will prove a more precise version.

Proposition 1. Let $k>0$. There exist $\delta, \sigma>0$ satisfying $\sigma+\delta<\pi$ such that if a compact connected $n$-dimensional Riemannian manifold $(\mathcal{M}, g)$ satisfies

$$
-k^{2} \leq K_{\mathcal{M}} \leq 1, \quad i_{\mathcal{M}} \geq \pi-\sigma, \quad V_{\mathcal{M}} \leq 3 V\left(\mathscr{B}_{\pi / 2-\sigma / 2}\right)+V\left(\mathscr{B}_{\delta / 2}\right),
$$

then $\mathcal{M}$ is homeomorphic to $S^{n}$.

Remark 1. The choice of $\sigma$ or $\delta$ here is of course not optimal. We conjecture that $\sigma<\pi / 2$ is optimal.

Proof of Proposition 1. We proceed by way of contradiction. Suppose there exists a manifold $M$ satisfying (1) that is not homeomorphic to $S^{n}$. Take points $p, q$ in $M$ such that $d(p, q)=d_{\mathcal{M}}$, the diameter $d_{\mathcal{M}}$ of $\mathcal{M}$. Then by a well-known topological fact (see for instance [Brown 1960]), there is a point $x_{0} \in \mathcal{M}-\mathbb{B}\left(p, i_{\mathcal{M}}\right) \cup \mathbb{B}\left(q, i_{\mathcal{M}}\right)$. Without loss of generality, let $d\left(q, x_{0}\right) \geq d\left(p, x_{0}\right)=l_{0}$. Therefore $l_{0} \geq i_{\mathcal{M}} \geq \pi-\sigma$. First we show an explicit upper bound on $d_{\mu}$.

Lemma 1. $d_{\mathcal{M}} \leq \pi-\sigma+\delta$.

Proof. We argue by contradiction. If $d_{\mu}>\pi-\sigma+\delta$, then we consider the balls $\mathbb{B}(p, \pi / 2-\sigma / 2+\delta / 2), \mathbb{B}(q, \pi / 2-\sigma / 2+\delta / 2)$ and $\mathbb{B}\left(x_{0}, l_{0}-\pi / 2+\sigma / 2-\delta / 2\right)$. They are obviously pairwise disjoint. Therefore since $K_{\mathcal{M}} \leq 1$, Günther's volume comparison theorem gives

$$
V_{\mathcal{M}} \geq 2 V\left(\mathscr{乃}_{\pi / 2-\sigma / 2+\delta / 2}\right)+V\left(\Re_{l_{0}-\pi / 2+\sigma / 2-\delta / 2}\right) .
$$

In what follows, we check that

$$
2 V\left(\Re_{\pi / 2-\sigma / 2+\delta / 2}\right)+V\left(\Re_{l_{0}-\pi / 2+\sigma / 2-\delta / 2}\right)>3 V\left(\mathscr{B}_{\pi / 2-\sigma / 2}\right)+V\left(\mathscr{B}_{\delta / 2}\right) .
$$

Noting that $l_{0}-\pi / 2+\sigma / 2-\delta / 2 \geq \pi / 2-\sigma / 2-\delta / 2>0$, we have

$$
V\left(\mathscr{B}_{l_{0}-\pi / 2+\sigma / 2-\delta / 2}\right) \geq V\left(\mathscr{乃}_{\pi / 2-\sigma / 2-\delta / 2}\right)
$$

By the definition of $S^{n}$, we have $V\left(\mathscr{B}_{r}\right)=\omega_{n-1} \int_{0}^{r}(\sin t)^{n-1} \mathrm{~d} t$ for any $r>0$, where $\omega_{n-1}$ is the volume of the standard unit $(n-1)$-sphere $S^{n-1}$. Since $\sin t$ is 
increasing in $(0, \pi / 2)$, we have

$$
\begin{aligned}
& \frac{1}{\omega_{n-1}}\left[2 V\left(\mathscr{B}_{\pi / 2-\sigma / 2+\delta / 2}\right)+V\left(\mathscr{B}_{l_{0}-\pi / 2+\sigma / 2-\delta / 2}\right)-3 V\left(\mathscr{B}_{\pi / 2-\sigma / 2}\right)-V\left(\mathscr{\Re}_{\delta / 2}\right)\right] \\
& \geq 2 \int_{0}^{\pi / 2-\sigma / 2+\delta / 2}(\sin t)^{n-1} \mathrm{~d} t+\int_{0}^{\pi / 2-\sigma / 2-\delta / 2}(\sin t)^{n-1} \mathrm{~d} t \\
& -3 \int_{0}^{\pi / 2-\sigma / 2}(\sin t)^{n-1} \mathrm{~d} t-\int_{0}^{\delta / 2}(\sin t)^{n-1} \mathrm{~d} t \\
& =\int_{\pi / 2-\sigma / 2}^{\pi / 2-\sigma / 2+\delta / 2}(\sin t)^{n-1} \mathrm{~d} t-\int_{\pi / 2-\sigma / 2-\delta / 2}^{\pi / 2-\sigma / 2}(\sin t)^{n-1} \mathrm{~d} t \\
& +\int_{\pi / 2-\sigma / 2}^{\pi / 2-\sigma / 2+\delta / 2}(\sin t)^{n-1} \mathrm{~d} t-\int_{0}^{\delta / 2}(\sin t)^{n-1} \mathrm{~d} t \\
& >\int_{\pi / 2-\sigma / 2}^{\pi / 2-\sigma / 2+\delta / 2}(\sin t)^{n-1} \mathrm{~d} t-\int_{\pi / 2-\sigma / 2-\delta / 2}^{\pi / 2-\sigma / 2}(\sin t)^{n-1} \mathrm{~d} t>0 .
\end{aligned}
$$

Clearly, the estimates (2) and (3) contradict the assumptions (1).

Lemma 2. If $\delta>0$ and $\sigma=2 / 3 \int_{0}^{\delta / 2}(\sin t)^{n-1} \mathrm{~d} t$ satisfy $\sigma+\delta<\pi$, then

$$
V\left(\mathscr{B}_{\delta / 2}\right)+V\left(\mathscr{B}_{\pi / 2-\sigma / 2}\right)>\frac{3}{2} V_{S^{n}} .
$$

Proof. In fact, since $|\sin t| \leq 1$,

$$
\begin{aligned}
V\left(\mathscr{B}_{\delta / 2}\right) & =\omega_{n-1} \int_{0}^{\delta / 2}(\sin t)^{n-1} \mathrm{~d} t=\frac{3}{2} \omega_{n-1} \sigma \\
& >3 \omega_{n-1} \int_{\pi / 2-\sigma / 2}^{\pi / 2}(\sin t)^{n-1} \mathrm{~d} t \\
& =3 V\left(\mathscr{B}_{\pi / 2}\right)-V\left(\mathscr{\Re}_{\pi / 2-\sigma / 2}\right)=\frac{3}{2} V_{S^{n}}-V\left(\mathscr{\Re}_{\pi / 2-\sigma / 2}\right) .
\end{aligned}
$$

Lemma 3. There exists a point $E$ on $\partial \mathbb{B}(p, \pi / 2-\sigma / 2)$, that is, the boundary of $\overline{\mathbb{B}}(P, \pi / 2-\sigma / 2)$, such that

$$
d(E, q) \leq \pi / 2-\sigma / 2+\delta \quad \text { and } \quad d\left(E, x_{0}\right) \leq l_{0}-\pi / 2+\sigma / 2+\delta .
$$

Proof. Since $i_{\mathcal{M}} \geq \pi-\sigma$, the boundary $\partial \mathbb{B}(p, \pi / 2-\sigma / 2)$ is arc-connected in $M$. Let $W=\gamma_{p, x_{0}} \cap \partial \mathbb{B}(p, \pi / 2-\sigma / 2)$ and $T=\gamma_{p, q} \cap \partial \mathbb{B}(p, \pi / 2-\sigma / 2)$. Take a continuous curve $f(t)(t \in 0,1])$ on $\partial \mathbb{B}(p, \pi / 2-\sigma / 2)$ such that $W=f(0)$ and $T=f(1)$. Let $\Gamma$ be the image curve of $f$, and let

$$
\begin{aligned}
& \Gamma_{1}=\{x \in \Gamma \mid d(x, q) \leq \pi / 2-\sigma / 2+\delta\}, \\
& \Gamma_{2}=\left\{x \in \Gamma \mid d\left(x, x_{0}\right) \leq l_{0}-\pi / 2+\sigma / 2+\delta\right\} .
\end{aligned}
$$

It is clear that $\Gamma_{1}$ and $\Gamma_{2}$ both are nonempty closed since $T \in \Gamma_{1}$ and $W \in \Gamma_{2}$. We will prove that there exists a point $E$ on $\Gamma$ satisfying (5). For this, we need only to 
verify that $\Gamma_{1} \cap \Gamma_{2} \neq \varnothing$. First we shall exclude the case that there exists a point $E$ in $\Gamma$ such that

$$
d(E, q)>\pi / 2-\sigma / 2+\delta \quad \text { and } \quad d\left(E, x_{0}\right)>l_{0}-\pi / 2+\sigma / 2+\delta .
$$

In fact, if (6) occurs, there must exist a point $F$ in the shortest geodesic $\bar{\gamma}_{p}$ issuing from $p$ and passing through $E$, such that $d(F, p)=\pi / 2-\sigma / 2+\delta / 2$. By the triangle inequality, we have

$$
\begin{aligned}
d(F, q) & \geq d(E, q)-d(E, F)>\pi / 2-\sigma / 2+\delta / 2, \\
d\left(F, x_{0}\right) & \geq d\left(E, x_{0}\right)-d(E, F)>l_{0}-\pi / 2+\sigma / 2+\delta / 2 .
\end{aligned}
$$

Therefore the four balls $\mathbb{B}(p, \pi / 2-\sigma / 2), \mathbb{B}(q, \pi / 2-\sigma / 2), \mathbb{B}\left(x_{0}, l_{0}-\pi / 2+\sigma / 2\right)$ and $\mathbb{B}(F, \delta / 2)$ are pairwise disjoint. Applying again Günther's volume comparison theorem, we get

$$
\begin{aligned}
V_{\mathcal{M}}>V(\mathbb{B}(p, \pi / 2-\sigma / 2))+V(\mathbb{B}(q, \pi / 2-\sigma / 2)) & \quad+V\left(\mathbb{B}\left(x_{0}, l_{0}-\pi / 2+\sigma / 2\right)\right)+V(\mathbb{B}(F, \delta / 2)) \\
& =2 V\left(\mathscr{B}_{\pi / 2-\sigma / 2}\right)+V\left(\mathscr{B}_{\pi / 2-\sigma / 2}\right)+V\left(\mathscr{B}_{\delta / 2}\right) \\
= & 3 V\left(\mathscr{B}_{\pi / 2-\sigma / 2}\right)+V\left(\mathscr{B}_{\delta / 2}\right),
\end{aligned}
$$

which contradicts the assumption on $V_{\mu}$. Thus (6) cannot hold, which means $\Gamma=\Gamma_{1} \cup \Gamma_{2}$. Since $\Gamma$ is connected, we get a point $E \in \Gamma_{1} \cap \Gamma_{2} \neq \varnothing$; this point clearly satisfies (5).

Lemma 1 and the triangle inequalities easily imply another result:

Corollary 1. The point E obtained in Lemma 3 satisfies the inequalities

$$
\begin{aligned}
& \pi / 2-\delta / 6<d(E, p)=\pi / 2-\sigma / 2, \\
& \pi / 2-\delta / 6 \leq d(E, q) \leq \pi / 2-\sigma / 2+\delta, \\
& \pi / 2-\delta / 6 \leq d\left(E, x_{0}\right) \leq l_{0}-\pi / 2+\sigma / 2+\delta .
\end{aligned}
$$

On the other hand,

$$
d(p, q) \leq \pi-\sigma+\delta \quad \text { and } \quad \pi-\sigma \leq l_{0}=d\left(p, x_{0}\right) \leq \pi-\sigma+\delta .
$$

Take $E \in \partial \mathbb{B}(p, \pi / 2-\sigma / 2)$ satisfying (5). We consider a geodesic triangle $\left(\gamma_{E, p}, \gamma_{E, x_{0}}, \gamma_{p, x_{0}}\right)$ in $\mu$. Since $K_{\mathcal{M}} \geq-k^{2}$, Toponogov's comparison theorem gives

(10) $\cosh \left[k d\left(p, x_{0}\right)\right]$

$$
\begin{aligned}
& \leq \cosh [k d(E, p)] \cosh \left[k d\left(E, x_{0}\right)\right]-\sinh [k d(E, p)] \sinh \left[k d\left(E, x_{0}\right)\right] \cos \alpha \\
& =\cosh \left[k\left(d(E, p)+d\left(E, x_{0}\right)\right)\right]-\sinh [k d(E, p)] \sinh \left[k d\left(E, x_{0}\right)\right](1+\cos \alpha),
\end{aligned}
$$


where the angle $\alpha$ is defined by $\alpha=\left.L\left(\dot{\gamma}_{E, p}, \dot{\gamma}_{E, x_{0}}\right)\right|_{E}$. By Corollary 1, we have

$$
\begin{aligned}
1+\cos \alpha & \leq \frac{\cosh \left(k\left(d(E, p)+d\left(E, x_{0}\right)\right)\right)-\cosh \left(k d\left(p, x_{0}\right)\right)}{\sinh (k d(E, p)) \sinh \left(k d\left(E, x_{0}\right)\right)} \\
& \leq \frac{\cosh \left(k\left(l_{0}+\delta\right)\right)-\cosh \left(k l_{0}\right)}{\sinh ^{2}(k(\pi / 2-\delta / 6))} .
\end{aligned}
$$

Clearly $t \mapsto \cosh (k(t+c))-\cosh (k t)$ is increasing in $[0, \infty)$ for $c>0$, so we get

$$
\begin{aligned}
1+\cos \alpha & <\frac{\cosh (k(\pi+2 \delta))-\cosh (k(\pi+\delta))}{\sinh ^{2}(k(\pi / 2-\delta / 6))} \\
& <\frac{\cosh (k(\pi+3 \delta))-\cosh (k(\pi+\delta))}{\sinh ^{2}(k(\pi / 2-\delta / 6))} .
\end{aligned}
$$

Similarly, if we consider the geodesic triangle $\left(\gamma_{E, p}, \gamma_{E, q}, \gamma_{p, q}\right)$ and the angle $\beta=$ $\left.\angle\left(\dot{\gamma}_{E, p}, \dot{\gamma}_{E, q}\right)\right|_{E}$, we have

$$
\begin{aligned}
1+\cos \beta & \leq \frac{\cosh (k(d(E, p)+d(E, q)))-\cosh (k d(p, q))}{\sinh (k d(E, p)) \sinh (k d(E, q))} \\
& \leq \frac{\cosh (k(\pi-\sigma+\delta))-\cosh \left(k l_{0}\right)}{\sinh ^{2}(k(\pi / 2-\delta / 6))} \\
& \leq \frac{\cosh (k(\pi-\sigma+2 \delta))-\cosh (k(\pi-\sigma))}{\sinh ^{2}(k(\pi / 2-\delta / 6))} \\
& <\frac{\cosh (k(\pi+3 \delta))-\cosh (k(\pi+\delta))}{\sinh ^{2}(k(\pi / 2-\delta / 6))}
\end{aligned}
$$

Likewise, if we think of the geodesic triangle $\left(\gamma_{E, q}, \gamma_{E, x_{0}}, \gamma_{q, x_{0}}\right)$ and the angle $\gamma=\left.\angle\left(\dot{\gamma}_{E, q}, \dot{\gamma}_{E, x_{0}}\right)\right|_{E}$, then, noting that $d\left(q, x_{0}\right) \geq l_{0} \geq \pi-\varepsilon_{0}$, we have

$$
\begin{aligned}
1+\cos \gamma & \leq \frac{\cosh \left(k\left(d(E, q)+d\left(E, x_{0}\right)\right)\right)-\cosh \left(k d\left(q, x_{0}\right)\right)}{\sinh (k d(E, q)) \sinh \left(k d\left(E, x_{0}\right)\right)} \\
& <\frac{\cosh (k(\pi+3 \delta))-\cosh (k(\pi+\delta))}{\sinh ^{2}(k(\pi / 2-\delta / 6))} .
\end{aligned}
$$

Now we will conclude the proof of Proposition 1 using the following lemma, whose proof will be postponed.

Lemma 4. For $k>0$, there exists a positive number $\delta_{0} \in(0,3 \pi / 5)$ such that $\delta_{0}$ is a solution of

$$
\cosh (k(\pi+3 t))-\cosh (k(\pi+t))-(1-\sqrt{3} / 2) \sinh ^{2}(k(\pi / 2-t / 6))=0 .
$$

Take $\delta=\delta_{0}$ in Lemma 4, take the $\sigma$ from Lemma 2, and let $E$ be the point given by Lemma 3. Obviously, $\sigma<\delta / 3$, hence $\sigma+\delta<4 \delta / 3<\pi$. Applying (12)-(14), 
one immediately deduces

$$
\cos \alpha<-\sqrt{3} / 2, \quad \cos \beta<-\sqrt{3} / 2, \quad \cos \gamma<-\sqrt{3} / 2 .
$$

That is,

$$
\alpha>2 \pi / 3, \quad \beta>2 \pi / 3, \quad \gamma>2 \pi / 3 .
$$

However, since $0 \leq \gamma \leq 2 \pi-(\alpha+\beta)$, we get a contradiction. Thus our hypothesis on $\mathcal{M}$ was wrong, so $\mathcal{M}$ must be homeomorphic to $S^{n}$.

In Theorem 1 or Proposition 1 , we require that the sectional curvature $K_{\mathcal{M}}$ is in the interval $\left[-k^{2}, 1\right]$ for some $k>0$. Trivially the result holds if $K_{\mu} \in(0,1]$. In the situation $0 \leq K_{\mathcal{M}} \leq 1$, we can simplify our proof by comparing against Euclidean space; however the estimates (12)-(14) would need to be changed for the case $k=0$.

Theorem 2. Suppose $(\mathcal{M}, g)$ is a compact connected $n$-dimensional Riemannian manifold with sectional curvature $0 \leq K_{\mathcal{M}} \leq 1$. Let $\delta>0$, and let

(16) $\sigma=\frac{2}{3} \int_{0}^{\delta / 2}(\sin t)^{n-1} \mathrm{~d} t \quad$ such that $(2-\sqrt{3})(\pi-\sigma)^{2}-16 \delta(\pi-\sigma+2 \delta) \geq 0$.

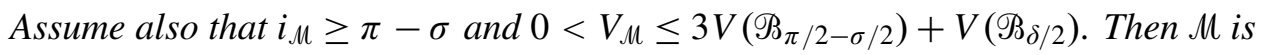
homeomorphic to $S^{n}$.

Proof. We prove this result by contradiction. If some manifold $\mathcal{M}$ satisfies the assumptions of Theorem 2 and is not homeomorphic to $S^{n}$, there is a point $x_{0} \in \mathcal{M}$ such that $x_{0} \in \mathcal{M}-\mathbb{B}\left(p, i_{\mathcal{M}}\right) \cup \mathbb{B}\left(q, i_{\mathcal{M}}\right)$, with $d(p, q)=d_{\mathcal{M}}$. Assume that $d\left(q, x_{0}\right) \geq$ $d\left(p, x_{0}\right)=l_{0} \geq i_{\mu}$. By Lemma 3, there exists a point $E \in \partial \mathbb{B}(p, \pi / 2-\sigma / 2)$ satisfying (5). By triangle inequality, we get because $K_{\mu} \geq 0$ that

$$
d(E, q) \geq \pi / 2-\sigma / 2 \quad \text { and } \quad d\left(E, x_{0}\right) \geq \pi / 2-\sigma / 2 .
$$

Now consider the geodesic triangle $\left(\gamma_{p, E}, \gamma_{x_{0}, E}, \gamma_{p, x_{0}}\right)$; let $\alpha=\left.L\left(\dot{\gamma}_{E, p}, \dot{\gamma}_{E, x_{0}}\right)\right|_{E}$. By Toponogov's comparison theorem,

$$
d^{2}\left(p, x_{0}\right) \leq d^{2}(E, p)+d^{2}\left(E, x_{0}\right)-2 d(E, p) d\left(E, x_{0}\right) \cos \alpha,
$$

so

$$
\begin{aligned}
1+\cos \alpha & \leq \frac{\left(d(E, p)+d\left(E, x_{0}\right)\right)^{2}-d^{2}\left(p, x_{0}\right)}{2 d(E, p) d\left(E, x_{0}\right)} \\
& \leq \frac{\left(l_{0}+\delta\right)^{2}-l_{0}^{2}}{2(\pi / 2-\sigma / 2)^{2}}<\frac{2 \delta(\pi-\sigma+2 \delta)}{(\pi / 2-\sigma / 2)^{2}}
\end{aligned}
$$


Similarly, consider the triangle $\left(\gamma_{E, p}, \gamma_{E, q}, \gamma_{p, q}\right)$, with $\beta=\left.\angle\left(\dot{\gamma}_{E, p}, \dot{\gamma}_{E, q}\right)\right|_{E}$ and the triangle $\left.\left(\gamma_{E, q}, \gamma_{E, x_{0}}, \gamma_{q, x_{0}}\right)\right)$, with $\gamma=\left.\angle\left(\dot{\gamma}_{E, q}, \dot{\gamma}_{E, x_{0}}\right)\right|_{E}$. Then

$$
\begin{aligned}
1+\cos \beta & \leq \frac{(d(E, p)+d(E, q))^{2}-d^{2}(p, q)}{2 d(E, p) d(E, q)} \\
& \leq \frac{(\pi-\sigma+\delta)^{2}-(\pi-\sigma)^{2}}{2(\pi / 2-\sigma / 2)^{2}}<\frac{2 \delta(\pi-\sigma+2 \delta)}{(\pi / 2-\sigma / 2)^{2}}, \\
1+\cos \gamma & \leq \frac{\left(d(E, q)+d\left(E, x_{0}\right)\right)^{2}-d^{2}\left(q, x_{0}\right)}{2 d(E, q) d\left(E, x_{0}\right)} \\
& \leq \frac{2 \delta\left(l_{0}+\delta\right)}{(\pi / 2-\sigma / 2)^{2}}<\frac{2 \delta(\pi-\sigma+2 \delta)}{(\pi / 2-\sigma / 2)^{2}} .
\end{aligned}
$$

Let $\delta$ and $\sigma$ satisfy (16). Then from (18) and (19), one can infer again that

$$
\alpha>2 \pi / 3, \quad \beta>2 \pi / 3, \quad \gamma>2 \pi / 3,
$$

which is impossible as above.

Proof of Lemma 4. First, we will show that the Equation (15) indeed contains a positive solution $\delta_{0}$. Define

$$
F(t, k)=\cosh (k(\pi+3 t))-\cosh (k(\pi+t))-(1-\sqrt{3} / 2) \sinh ^{2}(k(\pi / 2-t / 6)) .
$$

For fixed $k>0$ and for $t \in[0,3 \pi]$,

$$
\frac{d F}{d t}=k\left\{3 \sinh (k(3 t+\pi))-\sinh (k(t+\pi))+\frac{2-\sqrt{3}}{12} \sinh (k(\pi-t / 3))\right\}>0,
$$

which implies that $F(t, k)$ is increasing with respect to $t$ in $[0,3 \pi]$. Moreover, $F(0, k)<0$ and $F(3 \pi, k)>0$. So (15) has a unique solution $\delta_{0} \in(0,3 \pi)$ for any $k>0$. Consider the function $k \mapsto F(3 \pi / 5, k)$. Then

$$
\frac{d F}{d k}\left(\frac{3 \pi}{5}, k\right)=\frac{14 \pi}{5} \sinh \left(\frac{14 k \pi}{5}\right)-\frac{8 \pi}{5} \sinh \left(\frac{8 k \pi}{5}\right)-\frac{(2-\sqrt{3}) \pi}{5} \sinh \left(\frac{4 k \pi}{5}\right) .
$$

We can check that

$$
\frac{14 \pi}{5} \sinh \left(\frac{14 k \pi}{5}\right)-\frac{8 \pi}{5} \sinh \left(\frac{8 k \pi}{5}\right)>\frac{4 \pi}{5} e^{8 \pi / 5}>\frac{(2-\sqrt{3}) \pi}{5} \sinh \left(\frac{4 k \pi}{5}\right),
$$

which implies that $F(3 \pi / 5, k)$ is increasing in $[0, \infty)$. Note that $F(3 \pi / 5,0)=0$; thus $F(3 \pi / 5, k)>0$ for $k>0$. This shows there is a solution in $0<\delta_{0}<3 \pi / 5$.

\section{Acknowledgments}

I thank Professor Dong Ye, whose comments simplified many proofs of this paper. Also, I thank the referee for suggestions. 


\title{
References
}

[Brown 1960] M. Brown, "A proof of the generalized Schoenflies theorem", Bull. Amer. Math. Soc. 66 (1960), 74-76. MR 22 \#8470b Zbl 0132.20002

[Coghlan and Itokawa 1991] L. Coghlan and Y. Itokawa, "A sphere theorem for reverse volume pinching on even-dimensional manifolds", Proc. Amer. Math. Soc. 111:3 (1991), 815-819. MR 91f:53033 Zbl 0719.53019

[Grove and Shiohama 1977] K. Grove and K. Shiohama, "A generalized sphere theorem", Ann. Math. (2) 106:2 (1977), 201-211. MR 58 \#18268 Zbl 0341.53029

[Perelman 1995] G. Perelman, "A diameter sphere theorem for manifolds of positive Ricci curvature”, Math. Z. 218:4 (1995), 595-596. MR 96f:53056 Zbl 0831.53033

[Shen 1989] Z. M. Shen, "A sphere theorem for manifolds of positive Ricci curvature", Indiana Univ. Math. J. 38:1 (1989), 229-233. MR 90h:53044 Zbl 0678.53032

[Shiohama 1983] K. Shiohama, "A sphere theorem for manifolds of positive Ricci curvature", Trans. Amer. Math. Soc. 275:2 (1983), 811-819. MR 84c:53041 Zbl 0518.53047

[Suyama 1991] Y. Suyama, "Differentiable sphere theorem by curvature pinching", J. Math. Soc. Japan 43:3 (1991), 527-553. MR 92g:53033 Zbl 0790.53041

[Wen 2004] Y. Wen, "A note on pinching sphere theorem", C. R. Math. Acad. Sci. Paris 338:3 (2004), 229-234. MR 2005k:53040 Zbl 1046.53019

[Wu 1989] J. Y. Wu, "A diameter pinching sphere theorem for positive Ricci curvature", Proc. Amer. Math. Soc. 107:3 (1989), 797-802. MR 90h:53045 Zbl 0676.53045

Received October 5, 2008. Revised April 9, 2009.

\author{
YULIANG WEN \\ DEPARTMENT OF MATHEMATICS \\ EAST CHINA NORMAL UNIVERSITY \\ SHANGHAI 20062 \\ CHINA \\ ylwen@math.ecnu.edu.cn
}

